\title{
ANÁLISIS SUSTENTABLE DE LAS FINCAS DE BRÓCOLI (Brassica oleracea L. var. italica) en Santa Rosa de Quives, Lima, Perú
}

\section{SUSTAINABLE ANALYSIS OF BROCCOLI (Brassica oleracea L. var. italica) FARMS IN SANTA ROSA DE QUIVES, LIMA, PERÚ}

\author{
Cristina Karina Andrade Alvarado ${ }^{1}$
}

\begin{abstract}
Resumen
Se realizó un estudio en la provincia de Canta, (Lima, Perú), con el objetivo de caracterizar los sistemas de cultivo de brócoli y determinar la sustentabilidad de los mismos. Se confeccionaron indicadores de tipo económico, social y ecológico adecuados a los sistemas bajo estudio. La evaluación de la sustentabilidad mediante el empleo de indicadores permitió detectar de manera simple, clara y objetiva algunos puntos críticos de los sistemas de producción. Los resultados indican que el aspecto más crítico fue la dimensión ambiental con el indicador conservación de la vida del suelo porque no usan cobertura vegetal e incorporan poca materia orgánica al suelo. El indicador riesgo por contaminación mostró bajos índices por el uso de pesticidas de elevada toxicidad y mal manejo de envases vacíos. La dimensión económica resultó ser más sustentable a pesar de la alta dependencia de insumos externos. En el aspecto social, los indicadores más sustentables fueron calidad de vivienda, acceso a salud y servicios básicos. El uso de indicadores confirma que las fincas de brócoli no son sustentables. El desarrollo de indicadores es adecuado para detectar puntos críticos, establecer sus causas y proponer soluciones a largo plazo.
\end{abstract}

Palabras clave: sustentabilidad, brócoli, ambiente.

\begin{abstract}
A study was carried out in Canta, Lima (Peru), in order to characterize broccoli cropping systems to determine their sustainability.Economic, ecological and social indicators were prepared, according to the systems under study. Data analysis showed some critical points related to farm sustainability. The most critical component was the environmental one, according to the soil life conservation indicator, because they do not use plant cover and incorporate little organic matter. According to the scale, pollution risk indicator was low as a result of the application of pesticides of high toxicity and poor handling of empty containers. The economic dimension was more sustainable despite of the high dependence on external inputs. In the social aspect, predominant sustainable indicators were housing quality, access to health and basic services. Sustainability indicators confirm that broccoli farms are not sustainable. Indicators are adequate to detect critical sustainability aspects and to understand their causes to propose long term solutions.
\end{abstract}

Key words: sustainability, broccoli, environment.

\section{Introducción.}

En los últimos años, la creciente conciencia sobre el negativo impacto ambiental, social y cultural de ciertas prácticas de la agricultura moderna ha llevado a plantear la necesidad de un cambio hacia un modelo agrícola más sustentable (Gliessman, 2002). La producción hortícola en el valle de Chillón se ha distinguido y mantenido por aplicar los principios de la revolución verde en lo referente a la tecnología en el manejo de cultivos.

La aplicación de prácticas convencionales de manejo de cultivos que degradan los recursos naturales, han ocasionados múltiples consecuencias, entre ellas: contaminación de recursos naturales (suelo y agua), efectos nocivos e irreversibles sobre la salud del trabajador rural y su familia, del consumidor, de la población y del ambiente en general (Altieri, 2002).

Los análisis convencionales que se utilizan para determinar los resultados de los sistemas agropecuarios (por ejemplo, el de costo/beneficio) no son adecuados para evaluar su funcionamiento a largo plazo, ya que no integran al análisis las dimensiones sociales y ambientales, las cuales adquieren cada día mayor relevancia (Sarandón et al., 2004).

Para el presente estudio, los indicadores se construyeron de acuerdo a la metodología y el marco conceptual propuesto por Sarandón \& Flores (2009) y el resultado de investigaciones continuas con experiencias recogidas de sistemas de producción agrícola. La incipiente información sobre la sustentabilidad de los sistemas agrícolas, muestra la 
importancia de este trabajo de investigación. Finalmente, se plantea la hipótesis: las fincas productoras de brócoli del distrito de Santa Rosa de Quives son sustentables. Para comprobar la hipótesis se requiere de la construcción y aplicación de indicadores determinantes para la sustentabilidad ambiental, económica y social. Los objetivos del presente estudio fueron: caracterizar y evaluar la sustentabilidad de las fincas de brócoli en el distrito de Santa Rosa de Quives, Lima, Perú. Los objetivos específicos están orientados a identificar los puntos críticos y fortalezas, que afectan la sustentabilidad del sistema de producción.

\section{Materiales y métodos.}

El estudio se realizó en el distrito de Santa Rosa de Quives, provincia de Canta, departamento de Lima, Perú. Geográficamente se ubica entre los paralelos $2^{\circ} 76^{\prime}$ y $2^{\circ} 96^{\prime}$ Latitud sur y los meridianos $86^{\circ} 88^{\prime}$ y $87^{\circ}$ $12^{\prime}$ Longitud oeste con una altitud de $800 \mathrm{msnm}$. El promedio de precipitación anual es de $19.48 \mathrm{~mm}$, la temperatura media anual es de $20.7^{\circ} \mathrm{C}$ (SENAMHI, 2014). Se trabajó con los centros poblados Macas y Zapan, que tiene un total de 188 productores de brócoli. Los procedimientos metodológicos para el desarrollo de la investigación son los que a continuación se describen:

a).- Caracterización: Consistió en la descripción y análisis de variables seleccionadas inherentes a la producción agropecuaria de la zona. La información se recopiló mediante encuestas estructuradas, entrevistas y visitas a las parcelas. En la estimación de la muestra se utilizó el "Método de proporciones", metodología propuesta por INCAGRO, recomendada por Julca et al. (2009) y citada en Merma \& Julca (2011). Se encuestó a 65 agricultores responsables de la toma de decisiones en las fincas de brócoli, de un universo de 188 productores de la zona en estudio. La información obtenida fue organizada en una base de datos para su respectiva tabulación y evaluación, de acuerdo a los objetivos de la investigación. La información captada a través de la encuesta se codificó en el paquete Microsoft Excel.

b).- Selección de indicadores y cuantificación: Con el fin de identificar los puntos críticos que afectan a la sustentabilidad, fue efectuada la selección de indicadores mediante el criterio bottom up (de abajo a arriba), producto de una caracterización previa de los sistemas en estudio (Galván-Miyoshi et al., 2008) y el modelo de indicadores PER (Presión, Estado y Respuesta), que es explicado por efectos de causalidad, aplicado por Sarandón \& Flores (2009). Bajo este contexto, los indicadores de presión se expresan en la diversificación de los sistemas de cultivo, rendimiento, consumo y comercialización; los indicadores de respuesta están relacionados con agroforestería (uso de especies arbustivas y arbóreas en la parcela), manejo integrado de plagas y restitución de nutrientes con abonos orgánicos; y los indicadores de estado se corresponden con el área destinado a la producción de brócoli, disponibilidad de mano de obra y satisfacción de servicios básicos. Estos indicadores fueron agrupados de acuerdo a su naturaleza o comportamiento ambiental, económico y social, como se indica en la Tabla 1. Los indicadores, según Belcher et al. (2004), son importantes porque permiten medir la sustentabilidad de un sistema complejo. Estos fueron estandarizados en una escala numérica ( 0 a 4 ) donde, el valor más bajo (0) representa una situación crítica para la sustentabilidad y el valor más alto (4) una mayor sustentabilidad, según el grado de afectación o contribución a la sustentabilidad (Tabla 1). Metodológicamente, la estandarización facilita la comparación entre las unidades de producción; así como el análisis de las dimensiones ambiental, económica y social de la sustentabilidad (Sarandón et al., 2006). La ponderación consistió en multiplicar el valor estandarizado por un factor, solo para aquellos indicadores que contribuyen significativamente al fortalecimiento de la sustentabilidad económica; y como tales adquieren un valor diferenciado (Tabla 1). La selección de indicadores a ponderar está relacionada con incremento en el rendimiento, mejora del suelo por aporte de nutrientes, estrategia para reducir pérdidas de cultivos por situaciones ambientales adversas, reducción del riesgo económico, entre otros.

Se estimó la sustentabilidad general de las fincas de brócoli en el distrito de Santa Rosa de Quives (Lima, Perú) promediando los valores obtenidos para las tres dimensiones de análisis como se muestra en la Tabla 2.

Tabla 2: Fórmulas utilizadas para estimar la sustentabilidad por dimensiones de análisis para el distrito de Santa Rosa de Quives.

\section{Sustentabilidad Ambiental}

$$
=\left[\frac{(a 1+a 2+a 3)}{3}+\frac{(2 \mathrm{~b} 1+\mathrm{b} 2+\mathrm{b} 3)}{4}+\frac{(\mathrm{c} 1+\mathrm{c} 2)}{2}+\frac{(\mathrm{d} 1+\mathrm{d} 2)}{2}\right] / 4
$$

\section{Sustentabilidad Economica}

$$
=\frac{\left[2 a+\frac{(b 1+b 2+b 3)}{3}\right]}{3}
$$

\section{Sustentabilidad Social}

$$
\begin{gathered}
=\left[\frac{\left(\frac{2((a 1+2 a 2+2 a 3)+2 a 4)}{7}+2 \mathrm{~b}+\mathrm{c}+\mathrm{d}\right]}{6}\right] \\
\text { Sustentabilidad General }=\frac{S A+S E+S S}{3}
\end{gathered}
$$


Tabla 3: Resultados para la caracterización de las fincas productoras de brócoli en el distrito de Santa Rosa de Quives.

\begin{tabular}{|c|c|c|}
\hline Aspectos Ambientales & Aspectos Económicos & Aspectos Sociales \\
\hline $\begin{array}{l}\text { Cultivares de brócoli: } \\
\text { Imperial }(97 \%), \\
\text { Pirata }(3 \%) . \\
\text { Pendiente predominante: } \\
100 \%(<5 \%)\end{array}$ & $\begin{array}{l}\text { Área de producción } \\
\text { de Brócoli: } \\
38 \% 3-5 \mathrm{ha}, \\
42 \% 1-3 \mathrm{ha}, \\
5 \%>1 \mathrm{ha},\end{array}$ & $\begin{array}{l}\text { Calidad de Vivienda: } \\
83 \% \text { material noble, } \\
12 \% \text { adobe, } \\
5 \% \text { madera. } \\
\text { Servicios Básicos: }\end{array}$ \\
\hline $\begin{array}{l}\text { Incorporación de tipos de } \\
\text { materia orgánica: } \\
\text { 31\% Residuos, } \\
\text { 46\% residuos y estiércol, } \\
\text { 14\% residuos, compost y estiércol. } \\
\text { Tipo de labranza: }\end{array}$ & $\begin{array}{l}15 \% 5-10 \text { ha. } \\
\text { Rendimiento } \\
\text { de Brócoli: } \\
62 \% 12-14 \mathrm{tn}, \\
23 \% 14-16 \mathrm{tn}, \\
8 \%>16 \mathrm{tn} .\end{array}$ & $\begin{array}{l}46 \% \text { con } 4 \text { servicios, } \\
31 \% 3 \text { servicios, } \\
15 \% 2 \text { servicios. } \\
\text { Acceso a Salud: } \\
77 \% \text { médicos permanentes, } \\
23 \% \text { personal temporal. }\end{array}$ \\
\hline $\begin{array}{l}100 \% \text { tractor, rastra más de } 2 \\
\text { pasadas. } \\
\text { Rotación de cultivos: } \\
55 \% \text { cada campaña, } \\
28 \% \text { cada } 2 \text { campañas, } \\
17 \% \text { cada } 3 \text { campañas. }\end{array}$ & $\begin{array}{l}\text { Ingreso Mensual: } \\
46 \% \$ 307-\$ 368, \\
31 \% \$ 368--\$ 460 \\
15 \%>\$ 460 \\
8 \%<\$ 368 \\
\text { Canales }\end{array}$ & $\begin{array}{l}\text { Nivel de Instrucción: } \\
46 \% \text { secundario, } \\
28 \% \text { primario, } \\
12 \% \text { técnico. }\end{array}$ \\
\hline $\begin{array}{l}\text { Manejo de la cobertura vegetal: } \\
100 \%<10 \% \text { sup. Cubierta } \\
\text { Uso y Manejo de pesticidas: } \\
54 \% \text { pesticidas clase II, } \\
46 \% \text { pesticidas clase I. } \\
\text { Destino de los envases vacíos } \\
\text { de Agroquímicos: } \\
38 \% \text { acopia y hace el triple lavado, } \\
37 \% \text { no acopia, no el triple lavado, } \\
19 \% \text { hace el triple lavado, no acopia. }\end{array}$ & $\begin{array}{l}\text { Comercialización: } \\
40 \% 5 \text { canales, } \\
30 \% 4 \text { canales, } \\
20 \% 2 \text { canales. } \\
\text { Diversificación Agrícola: } \\
62 \% 4-5 \text { productos, } \\
38 \%>6 \text { productos. } \\
\text { Dependencia de Insumos } \\
\text { Externos: } \\
86 \%<10 \% \text { insumos, } \\
14 \% 10-20 \% \text { insumos. }\end{array}$ & $\begin{array}{l}46 \% \text { medianamente } \\
\text { satisfecho, } \\
43 \% \text { satisfecho, } \\
6 \% \text { muy satisfecho. } \\
\text { Participación en org. } \\
83 \% 1 \text { organización, } \\
12 \% 2 \text { organizaciones, } \\
5 \% 3 \text { organizaciones. } \\
\text { Conciencia Ecológica : } \\
46 \% \text { bajo, } \\
31 \% \text { nulo, } \\
23 \% \text { medio. }\end{array}$ \\
\hline
\end{tabular}

\section{Resultados y discusión.}

1.- Caracterizacion de las fincas de brócoli.

En el aspecto social.

El nivel de instrucción de los productores de brócoli es muy variable (Tabla 3 ). Similares resultados obtuvo el INEI (2012), que señaló que en el distrito de Santa Rosa de Quives el mayor número de productores agropecuarios tienen educación secundaria. El nivel de educación es un dato relevante que permite conocer el grupo objetivo con el que se está trabajando y con el que se va implementar medidas correctivas. En función de los puntos críticos identificados para el sistema y con la metodología apropiada, un $83 \%$ de los productores de brócoli posee vivienda con paredes de ladrillo y pisos de cemento. El $46 \%$ posee acceso a servicios básicos para las personas que viven y trabajan en él (electricidad, agua potable, telefonía celular y saneamiento sanitario). La satisfacción de los servicios básicos es un indicador importante para la sustentabilidad socio-económica por constituir una estrategia de prevención de enfermedades; y consecuentemente, por reducir gastos derivados de una atención médica. Además, se puede observar que el $100 \%$ de los encuestados tiene un centro médico en la zona de estudio (cercana a la finca en un radio de $5 \mathrm{~km}$ ). En cuanto a la satisfacción respecto de su actividad como productor de brócoli, en general, se mostraron entre medianamente satisfechos (46\%) y satisfechos $(43 \%)$. También se observa que el $83 \%$ pertenece a una organización (comité de regantes), seguido por un 12\% que pertenece a dos organizaciones (asociación de productores ganaderos y comité de regantes), y el 5\% pertenece a tres organizaciones (asociaciones del estado, asociación de productores ganaderos, comité de regantes). No existe asociación de productores hortícolas. Las actividades de estas instituciones están ligadas al ámbito de la producción, el comercio, las finanzas y las prestaciones de servicios, todo ello con la finalidad de mejorar las condiciones de vida del agricultor (Gómez, 2004). Entre los servicios que brinda una organización agraria está la de extensión rural. Esta es una labor muy importante que realizan los profesionales e instituciones en su conjunto, puesto que la extensión agrícola es un tipo de educación no formal que tiene como intención promover el conocimiento, en este caso agrícola, para poder facilitar el desarrollo de los agricultores (Mc Leod, 2003).

En el aspecto económico.

Se aprecia que en el distrito de Santa Rosa de Quives predominan las pequeñas unidades agropecuarias, aquellas que representan hasta 10 ha (FAO, 2010) con un $96.8 \%$ del total de productores. El número de pequeñas unidades agropecuarias se incrementó en un 40\% desde 1994 hasta 2012 representando el $81.8 \%$ (INEI, 2013). Los resultados de 
rendimiento por ha coinciden con lo reportado por la DRAL (2014) pues el promedio está en 12.2 tn/ha para el cultivo de brócoli. De lo anterior, se infiere que el potencial de rendimiento del cultivo de brócoli es alto aun cuando las condiciones de manejo no son las adecuadas (Tabla 3).

La comercialización de brócoli se realiza en los mercados mayoristas: Santa Anita, Unicachi, Huamantanga, Cumbre ubicado en el km 22 de la av. Túpac Amaru, a una distancia de $64 \mathrm{kms}$ del distrito de Santa Rosa de Quives), minoristas y a través de intermediarios, quienes fijan los precios de mercado en chacra de acuerdo a los comportamientos de los precios del mercado. El $62 \%$ tienen cuatro a cinco tipos de productos para ofertar en el mercado; seguido del $38 \%$, que oferta más de 6 tipos de producto. Según Nicholls et al. (2013), la diversificación de cultivos representa una estrategia a largo plazo para los agricultores que están experimentando un clima errático. El uso de la diversificación al interior de los sistemas agrícolas puede reducir en gran medida la vulnerabilidad de los sistemas de producción al mismo tiempo que protege a los agricultores rurales y a la producción agrícola.

Los sistemas pueden tener mayor autonomía y seguridad si generan sus insumos dentro del predio. Para ello se estimó la cantidad de entradas al sistema que pueden sustituirse con abonos verdes, estiércol, etc. Según la Tabla 3, un $86 \%$ de agricultores generan menos del $10 \%$ de los insumos dentro del predio y un $14 \%$ de productores obtienen entre 10 y $20 \%$ de sus insumos dentro del predio.

En el Aspecto Ambiental.

Destaca la predominancia de un único cultivar de brócoli. Según Nicholls et al. (2013), la disminución de la biodiversidad cultivada aumenta la susceptibilidad de los sistemas en relación a enfermedades, plagas y variaciones climáticas, y los convierte más dependientes de insumos externos. Por el contrario con el cultivo de varias especies se aumenta la diversidad genética, y con ello se favorece la biodiversidad. Alvarado (2006) indicó que existen implementos de mecanización al suelo que ayudan a descompactar los suelos y a prepararlos, con riesgos reducidos de erosión. Tales son los casos del subsolador, los arados de cinceles y los implementos de picos, púas o ganchos de vibración (vibrocultor). Según la Tabla 3, el 100\% de productores usan arado de discos, grada de discos, surcador, más de dos mecanizaciones en campo. La Tabla 3 muestra que el $55 \%$ de fincas hace rotaciones de cultivos cada campaña, un $28 \%$ rota cada 2 campañas, un $17 \%$ realiza rotaciones cada 3 campañas. Altieri (2002) considera que la rotación de cultivos entre las parcelas en algunos casos aporta nutrientes, provee de fuentes de materia orgánica diversa, y disminuye la incidencia de problemas sanitarios, entre otros aspectos. En este sentido, un sistema es sustentable a medida que disminuye el periodo de rotación a lo largo del tiempo. Todas las fincas tienen menos del $10 \%$ de la superficie del suelo cubierta. Se considera que un sistema es sustentable a medida que aumenta la cobertura del suelo con algún tipo de cultivo vegetal disminuyendo de este modo el impacto de las lluvias y el sol en la superficie y, por otro lado, aportando materia orgánica al suelo. Se reconoce que la presencia de vegetación circundante a los cultivos es muy importante ya que los ecosistemas naturales alojan a la biodiversidad (Gliessman, 2002). Se encontró que el $54 \%$ de pesticidas usados en la finca son de clase II (mediana toxicidad), y el $46 \%$ de pesticidas son de clase I (extrema toxicidad). Según Nicholls et al. (2013), el alto uso de pesticidas será responsable de la pérdida de biodiversidad del suelo al reducir la complejidad biológica, principalmente si éstos son de alta toxicidad. Además, los agroquímicos utilizados en el control de plagas son capaces de producir contaminación en suelos y aguas, tanto superficiales como subterráneas; y vegetación nativa, generando riesgo de intoxicación en seres vivos, incluyendo al hombre. Los resultados muestran que el $38 \%$ acopia pero no realiza el triple lavado, el $19 \%$ realiza el triple lavado pero no acopia, El $37 \%$ no realiza el triple lavado ni acopia.

Marañón (2015) señaló que los horticultores del valle del rio Chillón no aplican el triple lavado de los envases de los plaguicidas agrícolas de plástico rígidos. Los envases vacíos indican presencia de productos de contrabando y no cuentan con el registro para los cultivos en que son utilizados y son desechados de manera incorrecta. Además, carecen de infraestructura para el almacenamiento de los equipos de aplicación y protección, desecho y reciclaje de envases.

2.- Sustentabilidad de las fincas de brócoli.

Dimensión económica: Los resultados muestran que el $86.2 \%$ de las fincas evaluadas son económicamente sustentables $(\geq 2)$ y el $13.8 \%$ presenta una situación crítica para la sustentabilidad $(<2)$ pues son altamente dependientes de insumos externos. El ingreso neto mensual resulto favorable. El riesgo económico en general está bien manejado debido a la diversificación de la producción y tener más de 5 canales de comercialización.

Dimensión Ambiental: Los resultados indican que el $96.9 \%$ de las fincas evaluadas reflejan una situación crítica para la sustentabilidad ambiental, consecuencia del uso indiscriminado de pesticidas (clase I y II), inadecuado manejo de los envases vacíos de pesticidas, Además, no hay diversidad intraespecifica e interespecifica, y el riesgo de erosión es alto porque no existe un manejo sostenible del suelo. Según Nicholls et al. (2013), la disminución de la biodiversidad cultivada aumenta la susceptibilidad de los sistemas en relación a enfermedades, plagas y variaciones climáticas, y los convierte más dependientes de insumos externos. Por el contrario, con el cultivo de varias especies, se aumenta la diversidad genética, y con ello se favorece la biodiversidad. La 


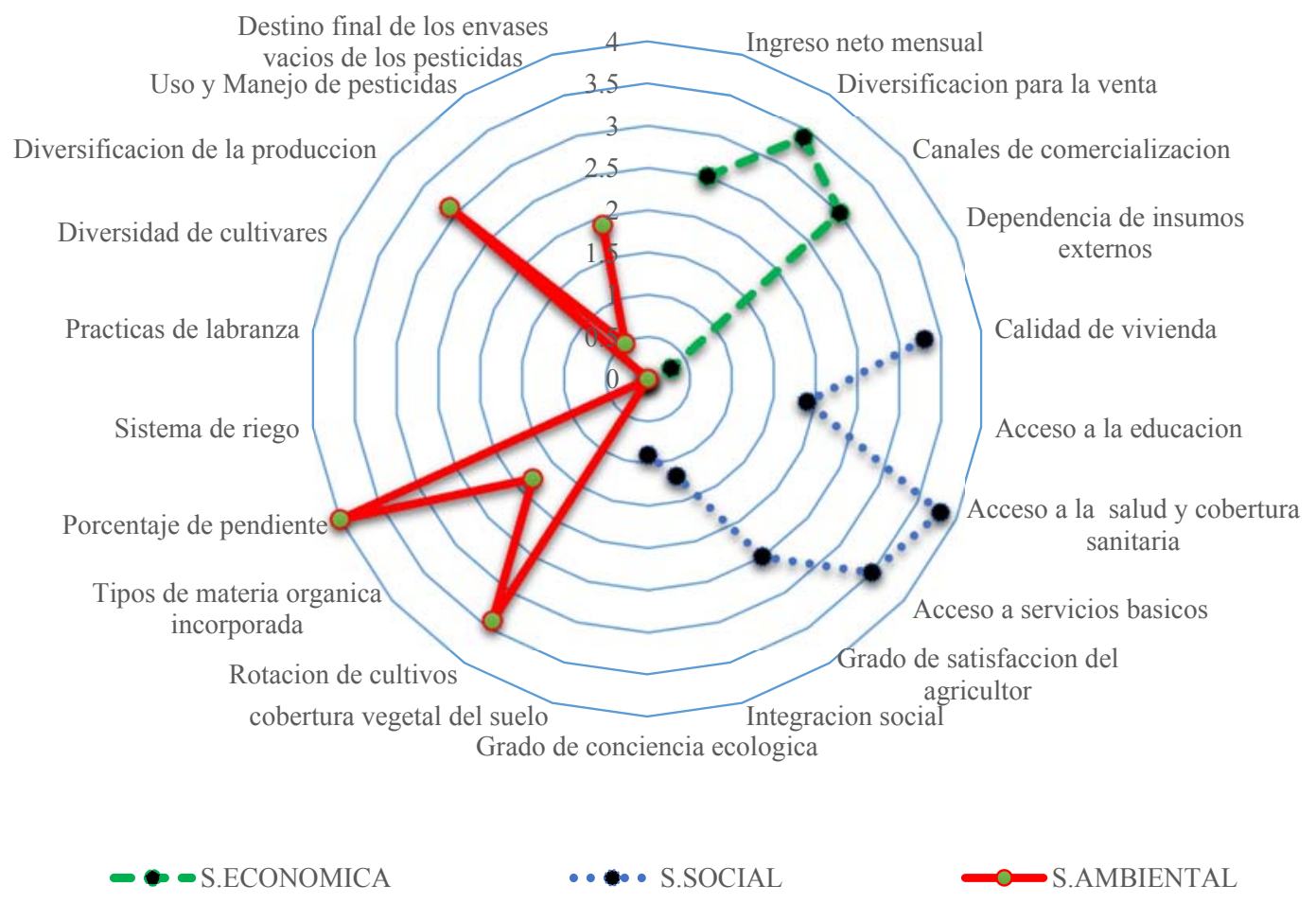

Figura 1: Puntos Críticos y sustentabilidad del sistema.

sustentabilidad ambiental aplicada a la agricultura está referida a la capacidad de garantizar la continuidad de la productividad agraria mediante el uso de prácticas en favor del uso adecuado de los recursos naturales (Gómez-Limón \& Arriaza, 2011).

Dimensión Social: Dentro de esta dimensión, el $80 \%$ de las fincas evaluadas son sustentables $(\geq 2)$, pues son sistemas bastante estables fundamentalmente porque tienen satisfechos sus servicios básicos, acceso a la educación y la salud que permite que los productores tengan un nivel de vida adecuado. Además, los indicadores que evaluaron el grado de aceptabilidad demostraron un mediano desarrollo de este aspecto. Sin embargo, los indicadores que evaluaron grado de integración social, conocimiento y conciencia ecológica fueron los más bajos. Existen también los indicadores culturales que recogen las prácticas tecnológicas locales. Estos indicadores del llamado "saber campesino" tratan de medir uno de los aspectos más complejos de la sostenibilidad de los sistemas de producción como lo es la generación de conocimientos a partir de sistemas distintos en escalas, propósitos, fuentes y usuarios (Glave \& Escobar, 2001). Los indicadores sociales están orientados a evaluar la satisfacción del productor, su calidad de vida y la integración social (Sarandón et al., 2006).

Sustentabilidad general: Como se ha señalado anteriormente, Sarandón (2002) propuso que para que una finca pueda ser considerada sustentable, el índice general (IGen.) debe ser mayor a 2; pero considerando que ninguno de los tres indicadores debe tener un valor menor a 2.

Los resultados muestran que el $3.1 \%$ de las fincas son sustentables $(\geq 2)$; mientras que $96.9 \%$ no son sustentables $(<2)$.

El índice de sustentabilidad general promedio de las 65 fincas fue 2.1. El manejo de las fincas satisfizo en mayor grado los objetivos económicos (2.4) que los objetivos socioculturales (2.2), o los objetivos ecológicos (1.6). Las condiciones de sustentabilidad se dan en un contexto de integración multidimensional donde se determinan las siguientes fortalezas del 
sistema de producción deseables de mantener: en el aspecto social tenemos a los servicios básicos satisfechos y aceptabilidad del sistema de producción; en el aspecto económico tenemos al ingreso neto mensual, diversificación para la venta y vías de comercialización; en el aspecto ambiental tenemos a la rotación de cultivos, diversificación de la producción. Los resultados obtenidos en esta investigación, confirman la utilidad de emplear un enfoque sistémico y holístico, con una óptica multicriterio para abordar la multidimensión de la sustentabilidad (Mendoza \& Prabhu, 2000).

Puntos críticos en las fincas de brócoli en Santa Rosa de Quives: Según las tres áreas de evaluación tenemos:

En el aspecto ambiental: Surgen seis puntos críticos (Figura 1). Uno referido al manejo de la biodiversidad, este es la nula biodiversidad intraespecifica en los predios. Dos referidos a la conservación de la vida del suelo: bajo contenido de materia orgánica y la nula cobertura vegetal. Uno que tiene que ver con el riesgo por erosión: las malas prácticas de labranza que utilizan. Dos referidos al riesgo de contaminación por el uso y manejo descuidado de los pesticidas (clase I, II) poniendo en peligro la salud de los trabajadores y de los consumidores generando externalidades negativas y poniendo en riesgo el ecosistema y el destino final de los envases vacíos.

En el aspecto económico: Según la Figura 1, hay un aspecto crítico en el sistema de producción: la alta dependencia de insumos externos (0.3) en las fincas. Por lo tanto, estas fincas serían rentables desde el punto de vista económico a corto plazo. Esto sugiere que la rentabilidad se estaría consiguiendo a costa del deterioro del capital natural y social.

En el aspecto Social: Hay dos aspectos críticos (Figura 1). Uno es el grado de conciencia ecológica, porque la mayoría de los encuestados no percibe las consecuencias que pueden ocasionar algunas prácticas de manejo. Otro es el grado de integración social. En este sentido, tienen poca participación en organizaciones, además, no existe asociación de productores hortícolas.

\section{Conclusiones.}

1.- La horticultura es una actividad intensiva que hace un alto uso de pesticidas. En específico, el sistema de producción de brócoli convencional es basado en un importante uso de pesticidas. Las fincas tienen un promedio de área de 1 a 5 ha, con un rendimiento promedio de brócoli en Santa Rosa de Quives de 12-16 Tn/ha. El $80 \%$ de las fincas tiene de 4 a 5 tipos de hortalizas. Las fincas utilizan más de 5 canales de comercialización, la mayoría de hogares tienen acceso a los servicios básicos como electricidad, acceso a la educación, agua y centro médico en la zona de estudio. Los productores emplean solo un cultivar predominante de brócoli, con nula cobertura vegetal, alta dependencia de insumos externos y pobre utilización de prácticas agrícolas sustentables.

2.- El $96.9 \%$ de las fincas productoras de brócoli no son sustentables ambiental, económica y socialmente. Por lo tanto, amerita la necesidad de cambio del sistema productivo actual para un modo más sostenible.

3.- Para que las fincas productoras de brócoli sean sustentables es necesario reducir los puntos críticos del sistema: En general , aumentando la biodiversidad intraespecifica en los predios, mejorando el contenido de materia orgánica, usando cobertura vegetal, utilizando prácticas de labranza que no erosionen el suelo, eliminando el uso de agroquímicos I y II, incentivando la organización y concientización de temas ambientales a los agricultores, etc- Valdría la pena realizar una conclusión priorizando los aspectos que las fincas deben gestionar para mejorar su sustentabilidad, i.e., reduciendo el uso de agroquímicos I y II, mejorando la cobertura de suelo, etc.

\section{Literatura citada.}

Altieri M. 2002. Agroecología: Bases científicas para una agricultura sustentable. Guaíba, BR. Agropecuaria, 592 p.

Alvarado CH. 2006. Método de labranza conservacionista y maquinaria para la conservación de los suelos. Taller de Publicaciones, Instituto Tecnológico de Costa Rica. Cartago. Vol. 19-1:56-60.

Belcher K.W., Boehm M.M. \& Fulton M.E. 2004. Agroecosystem Sustainability: A system simulation model approach. Agricultural systems 79(2):225-241.

DRAL (Dirección Regional de Agricultura Lima). 2014. Información agrícola: campaña agrícola 2014-2015. 20p.

FAO (Organización de las Naciones Unidas para la Agricultura y la Alimentación, IT). 2010. Los mercados mundiales de frutas y verduras orgánicas (en línea) Consultado 05 abr. 2014. Disponible en htpp://www.org/docrep/004y1669S/y1669S00.htm.

Galván-Miyoshi Y., Masera O. \& López-Ridaura S. 2008. Las evaluaciones de sustentabilidad. En: Astier M. Masera O. \& Galván-Miyoshi Y. Evaluación de Sustentabilidad. Un enfoque dinámico y multidimensional. Sec. 2: 41-57.

Gómez J. 2004. La Reforma de la PAC y la importancia de las cooperativas agrarias en la vertebración socioeconómica y territorial del medio rural. ERIA 63: 73-90.

Gómez-Limón J.A. \& Arriaza M. 2011. Evaluación de la sostenibilidad de las explotaciones de olivar en Andalucía. 40p.

Glave M. \& Escobar, J. 2001. Indicadores de sostenibilidad para la agricultura andina, proyecto: políticas integradas para el desarrollo rural sostenible-GDRUPA, En: Debate Agrario $\mathrm{N}^{\circ} 23,230 \mathrm{p}$

Gliessman S.R. 2002. Agroecología: procesos ecológicos en agricultura sostenible. Turrialba, C. R.: CATIE. P. 319329.

INEI (Instituto Nacional de Estadística e Información). 2012. IV Censo Agropecuario Lima, Perú, 20 p.

INEI (Instituto Nacional de Estadística e Información, Pe). 2013. XI Censo Nacional de Población y Vivienda, Lima, Perú. $180 \mathrm{p}$. 
Julca A., Meneses L., Rodriguez P., Bello S., Anahui J., Julca N., Borjas R., Crespo R., Santibañez R., Fundes G., Fundes D \& Reynoso A. 2009. Selección de fuentes naturales para la fertilización de café en el marco de una agricultura orgánica. [Comparación de Línea de base 2006 - Línea de Cierre, 2008]. Informe final de proyecto financiado por Incagro. Unalm-FDA.INIA-Café PerúJNC. Lima. 32p.

McLeod W. 2003. Agricultural Extension, Rural Development and teh Food Security Challenge. FAO. Italy. 35p.

Marañon P. 2015. Manejo y uso de plaguicidas agrícolas entre los horticultores en el valle del rio Chillón- Lima. Tesis Ing. Agr. Perú, Unalm. 154 p.

Mendoza G. \& Prabhu R. 2000. Multiple criteria decision making approaches to assessing forest sustainability using criteria and indicators: a case study. Forest Ecology and Management 131: 107-126

Merma I. \& Julca A. 2011. Evaluación y diseño de fincas en la selva alta bajo sistemas de cultivos prevalecientes en la
Convención-Cusco. UNALM. Rev. Ecol. Apl. Vol. 11(1): 1-11.

Nicholls CI., Rios L.A. \& Altieri M. 2013. Agroecología y resiliencia socio ecológica adaptándose al cambio climático. Colombia. Cyted. 2ed. p.18-29.

Sarandon S. J. 2002. El desarrollo y uso de indicadores para evaluar la sustentabilidad de los agroecosistemas. La Plata: Ediciones Científicas Americanas. (pp. 393-414).

Sarandon J., Zuluaga S., Cieza R., Gomez C., Janjetic L. \& Negrete E. 2004. Evaluación de la sustentabilidad de sistemas agrícolas de fincas en Misiones, Argentina, mediante el uso de indicadores. Agroecología, 1: 19-28.

Sarandon J. \& Flores C. 2009. Evaluación de la sustentabilidad en agroecosistemas: una propuesta metodológica. Agroecología 4:19 - 28

SENAMHI (Servicio Nacional de Meteorología e Hidrología, Pe). 2014. Atlas climático de precipitación y temperatura del aire en la cuenca del río Chillón.

Tabla 1: Indicadores de Sustentabilidad para el distrito de Santa Rosa de Quives según la metodología de Sarandón \& Flores (2009).

\begin{tabular}{|c|c|c|c|c|c|c|c|}
\hline \multirow{2}{*}{ 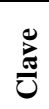 } & \multirow[b]{2}{*}{ Indicadores } & \multicolumn{5}{|c|}{ Escala de estandarización } & \multirow{2}{*}{$\begin{array}{l}\text { Factor } \\
\text { de } \\
\text { Pond. }\end{array}$} \\
\hline & & $\mathbf{0}$ & 1 & 2 & 3 & 4 & \\
\hline \multicolumn{8}{|c|}{ Dimensión Ambiental } \\
\hline a 1 & $\begin{array}{l}\text { \% cobertura } \\
\text { vegetal del } \\
\text { suelo }\end{array}$ & $<10 \%$ & $10 \%-30 \%$ & $30 \%-50 \%$ & $50 \%-70 \%$ & $>70 \%$ & 1 \\
\hline a2 & $\begin{array}{l}\text { Rotación de } \\
\text { cultivos }\end{array}$ & Ninguna & >3 campañas & $\begin{array}{l}\text { cada } 3 \\
\text { campañas }\end{array}$ & $\begin{array}{l}\text { cada } 2 \\
\text { campañas }\end{array}$ & $\begin{array}{l}\text { cada } \\
\text { campaña }\end{array}$ & 1 \\
\hline a3 & $\begin{array}{l}\text { Tipos de } \\
\text { Materia } \\
\text { orgánica } \\
\text { incorporados }\end{array}$ & Ninguno & 1 tipo & 2 tipos & 3 tipos & $>3$ tipos & 1 \\
\hline \multirow[t]{2}{*}{ b1 } & Porcentaje de & Más de & Entre & Entre & Entre & Inferior a & 2 \\
\hline & Pendiente & $20 \%$ & 15 y $20 \%$ & 10 y $15 \%$ & 5 y $10 \%$ & $5 \%$ & \\
\hline b2 & $\begin{array}{l}\text { Sistema de } \\
\text { riego }\end{array}$ & $\begin{array}{l}\text { Riego } \\
\text { surcos }\end{array}$ & $\begin{array}{l}\text { surcos y } \\
\text { aspersión }\end{array}$ & surcos y goteo & aspersión & $\begin{array}{l}\text { Riego por } \\
\text { goteo }\end{array}$ & 1 \\
\hline \multirow[t]{2}{*}{ b3 } & \multirow{2}{*}{$\begin{array}{l}\text { Prácticas de } \\
\text { labranza }\end{array}$} & $>\quad 2$ & una & \multirow{2}{*}{$\begin{array}{l}\text { Labranza con } \\
\text { fuerza animal }\end{array}$} & \multirow{2}{*}{$\begin{array}{l}\text { Labranza } \\
\text { mínima }\end{array}$} & \multirow{2}{*}{$\begin{array}{l}\text { Labranza } \\
\text { cero }\end{array}$} & \multirow[t]{2}{*}{1} \\
\hline & & mecanizaciones & mecanización & & & & \\
\hline $\mathbf{c 1}$ & $\begin{array}{l}\text { Diversidad de } \\
\text { cultivares }\end{array}$ & $1 \mathrm{cv}$ & $2 \mathrm{cv}$ & $3 \mathrm{cv}$ & $4 \mathrm{cv}$ & $>4 \mathrm{cv}$ & 1 \\
\hline c2 & $\begin{array}{l}\text { Diversificación } \\
\text { producción }\end{array}$ & 1 cultivo & 2 cultivos & 3 cultivos & 4 cultivos & $>4$ cultivos & 1 \\
\hline d1 & $\begin{array}{l}\text { Uso y manejo } \\
\text { de pesticidas }\end{array}$ & Clase I & clase II & Clase III & clase IV & No usa. & 1 \\
\hline d2 & $\begin{array}{l}\text { Destino final de } \\
\text { los envases } \\
\text { vacíos de los } \\
\text { pesticidas }\end{array}$ & $\begin{array}{lr}\text { Arroja } & \text { envases } \\
\text { vacíos } & \text { en } \\
\text { campo } & \end{array}$ & $\begin{array}{l}\text { No realiza el } \\
\text { triple lavado } \\
\text { No acopia }\end{array}$ & $\begin{array}{l}\text { Acopia pero no } \\
\text { realiza el triple } \\
\text { lavado }\end{array}$ & $\begin{array}{l}\text { Realiza el } \\
\text { triple lavado } \\
\text { pero no } \\
\text { acopia }\end{array}$ & $\begin{array}{l}\text { Acopia } \\
\text { después del } \\
\text { triple lavado }\end{array}$ & 1 \\
\hline \multicolumn{8}{|c|}{ Dimensión Económica } \\
\hline \multirow[t]{2}{*}{ a1 } & Ingreso neto & Menos de & Entre & Entre & Entre & Más de & \multirow[t]{2}{*}{2} \\
\hline & mensual & $\$ 230$ & $\$ 230-307$ & $\$ 307$ a $\$ 368$ & $\$ 368$ a $\$ 460$ & $\$ 460$ & \\
\hline b1 & $\begin{array}{l}\text { Diversificación } \\
\text { para la venta }\end{array}$ & 1 producto & 2 productos & 3 productos & 4-5 productos & $>6$ productos & 1 \\
\hline b2 & $\begin{array}{l}\text { Canales de } \\
\text { comercializac. }\end{array}$ & 1 canal & 2 canales & 3 canales & 4 canales & 5 canales & 1 \\
\hline b3 & $\begin{array}{l}\text { Dependencia de } \\
\text { insumos } \\
\text { externos } \\
\end{array}$ & $>40 \%$ & $30-40 \%$ & $20-30 \%$ & $10-20 \%$ & $<10 \%$ & 1 \\
\hline
\end{tabular}




\section{ANÁLISIS SUSTENTABLE DE FINCAS DE BRÓCOLI}

Julio - Diciembre 2017

Tabla 1: Indicadores de Sustentabilidad para el distrito de Santa Rosa de Quives según la metodología de Sarandón \& Flores (2009).

\begin{tabular}{|c|c|c|c|c|c|c|c|}
\hline \multirow{2}{*}{$\frac{\mathbb{Z}}{0}$} & \multirow[b]{2}{*}{ Indicadores } & \multicolumn{5}{|c|}{ Escala de estandarización } & \multirow{2}{*}{$\begin{array}{l}\text { Factor } \\
\text { de } \\
\text { Pond. }\end{array}$} \\
\hline & & $\mathbf{0}$ & 1 & 2 & 3 & 4 & \\
\hline \multicolumn{8}{|c|}{ Dimensión Social } \\
\hline a1 & $\begin{array}{l}\text { Calidad de } \\
\text { vivienda }\end{array}$ & Muy mala & Mala & Regular & Buena & Muy buena & 2 \\
\hline a2 & $\begin{array}{l}\text { Acceso a la } \\
\text { educación }\end{array}$ & Sin acceso & primaria & secundaria & técnico & universitario & 2 \\
\hline a3 & $\begin{array}{l}\text { Acceso a la } \\
\text { salud y } \\
\text { cobertura } \\
\text { sanitaria }\end{array}$ & $\begin{array}{l}\text { Sin centro } \\
\text { sanitario }\end{array}$ & $\begin{array}{l}\text { Centro de } \\
\text { Salud mal } \\
\text { equipado y } \\
\text { sin personal } \\
\text { idóneo }\end{array}$ & $\begin{array}{l}\text { Centro de } \\
\text { Salud mal } \\
\text { equipado y } \\
\text { personal } \\
\text { temporario }\end{array}$ & $\begin{array}{l}\text { Centro de } \\
\text { Salud } \\
\text { medianament } \\
\text { e equipado } \\
\text { con personal } \\
\text { temporario }\end{array}$ & $\begin{array}{l}\text { Centro de } \\
\text { Salud } \\
\text { Altamente } \\
\text { equipado y } \\
\text { médicos } \\
\text { permanentes }\end{array}$ & 2 \\
\hline $\mathrm{a} 4$ & $\begin{array}{l}\text { Acceso a } \\
\text { Servicios } \\
\text { básicos }\end{array}$ & 1 servicio & 2 servicios & 3 servicios & 4 servicios & $>4$ s. básicos & 2 \\
\hline $\mathrm{B}$ & $\begin{array}{l}\text { Grado de } \\
\text { satisfacción del } \\
\text { productor }\end{array}$ & Insatisfecho & $\begin{array}{l}\text { Poco } \\
\text { satisfecho }\end{array}$ & $\begin{array}{l}\text { Medianamente } \\
\text { satisfecho }\end{array}$ & Satisfecho & $\begin{array}{l}\text { Muy } \\
\text { satisfecho }\end{array}$ & 2 \\
\hline $\mathrm{C}$ & $\begin{array}{l}\text { Integración } \\
\text { social }\end{array}$ & Nula & Baja & media & Alta & Muy alta & 1 \\
\hline $\mathrm{D}$ & $\begin{array}{l}\text { Grado de } \\
\text { conciencia } \\
\text { ecológica }\end{array}$ & Nula & Baja & media & Alta & Muy alta & 1 \\
\hline
\end{tabular}

${ }^{1}$ Ing. Agrónoma. Universidad Nacional Agraria La Molina. Av. La Molina s/n La Molina Lima - Perú. Correo electrónico: cristykaa@hotmail.com. 